\begin{tabular}{|c|l|}
\hline Title & Tumor location affects the results of simple excision for multiple osteochondromas in the forearm. \\
\hline Author(s) & Ishikawa, Jun-ichi; Kato, Hiroyuki; Fujioka, Fumio; Iwasaki, Norimasa; Suenaga, Naoki; Minami, A kio \\
\hline Citation & $\begin{array}{l}\text { The Journal of Bone and Joint Surgery, 89(6), 1238-1247 } \\
\text { https://doi.org/L0.2106/JBJS.F.00298 }\end{array}$ \\
\hline Issue Date & 2007-06 \\
\hline Doc URL & http://hdl.handle.net/2115/28000 \\
\hline Type & article \\
\hline File Information & JBJS89-6.pdf \\
\hline
\end{tabular}

Instructions for use 
The Journal of Bone E Joint Surgery
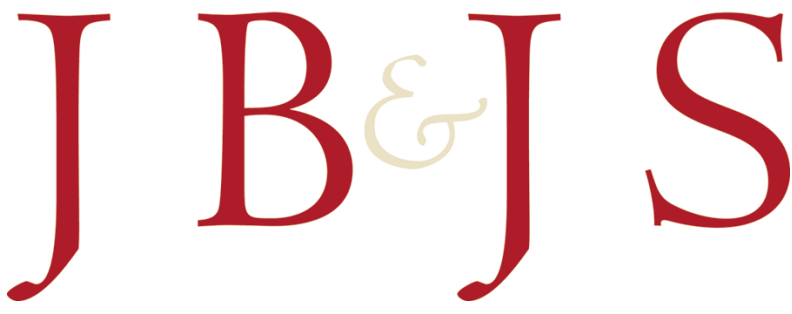

This is an enhanced PDF from The Journal of Bone and Joint Surgery

The PDF of the article you requested follows this cover page.

\section{Tumor Location Affects the Results of Simple Excision for Multiple Osteochondromas in the Forearm}

Jun-ichi Ishikawa, Hiroyuki Kato, Fumio Fujioka, Norimasa Iwasaki, Naoki Suenaga and Akio Minami J Bone Joint Surg Am. 2007;89:1238-1247. doi:10.2106/JBJS.F.00298

\section{This information is current as of August 8, 2007}

Reprints and Permissions

Click here to order reprints or request permission to use material from this article, or locate the article citation on jbjs.org and click on the [Reprints and Permissions] link.

Publisher Information

The Journal of Bone and Joint Surgery

20 Pickering Street, Needham, MA 02492-3157

www.jbjs.org 


\title{
Tumor Location Affects the Results of Simple Excision for Multiple Osteochondromas in the Forearm
}

\author{
By Jun-ichi Ishikawa, MD, Hiroyuki Kato, MD, Fumio Fujioka, MD, Norimasa Iwasaki, MD, \\ Naoki Suenaga, MD, and Akio Minami, MD \\ Investigation performed at the Department of Orthopaedic Surgery, Hokkaido University School of Medicine, Sapporo, Japan
}

\begin{abstract}
Background: The effectiveness of excision of osteochondromas in controlling the progression of forearm and wrist deformity remains an issue of controversy. The purpose of this study was to analyze the effectiveness of tumor excision in the correction of forearm and wrist deformity due to multiple osteochondromas in children, with an interpretation of the results based on different patterns of deformity.
\end{abstract}

Methods: Fourteen forearms in thirteen children with a follow-up of more than twenty-four months (average, fiftythree months) were included in the study. The forearms were divided into two groups on the basis of the location of the tumor and the pattern of deformity. In Group 1 (six forearms), the osteochondroma was only in the distal aspect of the ulna and caused compression of the radius. In Group 2 (eight forearms), tumors were in both the distal aspect of the ulna and the ulnar side of the distal part of the radius and were in contact with each other. Radial length, ulnar shortening, radial bowing, the radial articular angle, and carpal slip were measured as radiographic parameters. UInar shortening and radial bowing were expressed as a percentage of the radial length to make it possible to compare data between the individuals. Each parameter was evaluated before surgery and at the time of final follow-up.

Results: In Group 1, the percentage of ulnar shortening and the percentage of radial bowing had improved at the time of final follow-up; however, in Group 2, both the radial articular angle and the percentage of radial bowing had deteriorated significantly after the tumor excision ( $p=0.049$ and $p=0.017$, respectively), even though the percentage of ulnar shortening showed no change.

Conclusions: The effectiveness of simple excision of osteochondromas of the distal aspect of the forearm is influenced by the tumor location and is related to the pattern of the deformity. Simple tumor excision can correct the forearm deformity in patients with an isolated tumor of the distal part of the ulna. Conversely, in patients with tumors involving the distal part of the ulna and the ulnar side of the distal end of the radius, tumor excision alone is a less promising procedure for the correction of the deformity.

Level of Evidence: Prognostic Level IV. See Instructions to Authors for a complete description of levels of evidence.

$\mathrm{M}$ ultiple osteochondromas frequently affect the distal aspects of the radius and ulna and can result in severe deformity of the wrist and forearm. Such deformity is caused by a combination of shortening of the ulna, bowing of the radius, and ulnar deviation of the wrist, and, occasionally, radial head dislocation ${ }^{1}$. Radial articular deformity in association with multiple osteochondromas (an increasing radial articular angle) may be induced by tethering of the shortened distal aspect of the ulna, as first proposed by Solomon in $1961^{2}$, and subsequently supported by several other studies $^{3-5}$. Solomon also theorized that relative ulnar shortening was accommodated by either bowing of the radius or dislocation of the radial head proximally. However, more recently Burgess and Cates performed a radiographic evaluation of a forearm deformity in thirty-five patients (sixty-five forearms) with multiple osteochondromas and showed that ulnar short-

Disclosure: The authors did not receive any outside funding or grants in support of their research for or preparation of this work. Neither they nor a member of their immediate families received payments or other benefits or a commitment or agreement to provide such benefits from a commercial entity. No commercial entity paid or directed, or agreed to pay or direct, any benefits to any research fund, foundation, division, center, clinical practice, or other charitable or nonprofit organization with which the authors, or a member of their immediate families, are affiliated or associated. 
The JOURnAL of BOnE \& JOINT SURGERY · JBJS.ORG VOlume 89-A · Number 6 · June 2007
Tumor Location Affects the Results of Simple Excision For Multiple Osteochondromas in the Forearm

TABLE I Radiographic Data on All Patients Before the Operation and at the Time of the Final Follow-up

\begin{tabular}{|c|c|c|c|c|c|c|c|c|c|c|c|c|}
\hline \multirow[b]{2}{*}{ Case } & \multirow[b]{2}{*}{ Gender } & \multirow{2}{*}{$\begin{array}{c}\text { Age at Time } \\
\text { of Operation } \\
(y r+m o)\end{array}$} & \multirow[b]{2}{*}{ Side } & \multirow{2}{*}{$\begin{array}{l}\text { Duration of } \\
\text { Follow-up (mo) }\end{array}$} & \multicolumn{2}{|c|}{$\begin{array}{c}\text { Ulnar } \\
\text { Shortening (\%) }\end{array}$} & \multicolumn{2}{|c|}{$\begin{array}{c}\text { Radial } \\
\text { Bowing (\%) }\end{array}$} & \multicolumn{2}{|c|}{$\begin{array}{l}\text { Radial Articular } \\
\text { Angle (deg) }\end{array}$} & \multicolumn{2}{|c|}{$\begin{array}{c}\text { Carpal } \\
\text { Slip* (\%) }\end{array}$} \\
\hline & & & & & Preop. & Final & Preop. & Final & Preop. & Final & Preop. & Final \\
\hline \multicolumn{13}{|c|}{ Group 1} \\
\hline 1 & $\mathrm{~F}$ & $5+2$ & $\mathrm{R}$ & 25 & 7.7 & 8.4 & 6.9 & 6.2 & 29 & 28 & 100 & 83.3 \\
\hline 2 & $\mathrm{~F}$ & $9+2$ & $\mathrm{R}$ & 48 & 8.9 & 6.5 & 13.1 & 11.0 & 42 & 41 & 52.2 & 58.3 \\
\hline 3 & $\mathrm{~F}$ & $6+3$ & L & 77 & 6.7 & 2.9 & 7.6 & 3.9 & 26 & 26 & 53.8 & 45.5 \\
\hline 4 & $\mathrm{~F}$ & $4+1$ & L & 27 & 15.2 & 11.8 & 13.0 & 8.1 & 28 & 28 & - & - \\
\hline 5 & $\mathrm{~F}$ & $12+3$ & $\mathrm{R}$ & 24 & 2.5 & 1.9 & 10.3 & 8.8 & 38 & 36 & 75.0 & 57.1 \\
\hline 6 & M & $4+5$ & L & 97 & 9.0 & 7.4 & 10.4 & 5.8 & 20 & 21 & - & - \\
\hline \multicolumn{13}{|l|}{ Group 2} \\
\hline 7 & M & $6+2$ & L & 46 & 9.3 & 8.3 & 9.3 & 9.4 & 42 & 63 & 90.0 & 111.8 \\
\hline \multirow[t]{2}{*}{8} & M & $14+6$ & $\mathrm{R}$ & 24 & 0.3 & 0.3 & 6.2 & 7.9 & 47 & 41 & 93.3 & 70.6 \\
\hline & & & L & 24 & 0.3 & 1.9 & 5.8 & 8.3 & 35 & 55 & 76.9 & 105.9 \\
\hline 9 & $\mathrm{~F}$ & $5+4$ & L & 54 & 13.2 & 12.9 & 6.6 & 8.4 & 34 & 37 & 90.9 & 47.4 \\
\hline 10 & M & $8+11$ & $\mathrm{R}$ & 83 & 5.8 & 6.9 & 7.2 & 6.9 & 35 & 33 & 56.3 & 35.7 \\
\hline 11 & M & $9+4$ & L & 48 & 7.3 & 5.1 & 6.5 & 8.5 & 28 & 42 & 62.5 & 62.5 \\
\hline 12 & $\mathrm{~F}$ & $7+1$ & $\mathrm{R}$ & 92 & 2.9 & 3.0 & 5.9 & 7.5 & 32 & 42 & 45.5 & 56.0 \\
\hline 13 & $M$ & $9+10$ & $\mathrm{R}$ & 77 & 4.4 & 7.7 & 7.3 & 7.3 & 23 & 28 & 57.1 & 33.3 \\
\hline
\end{tabular}

ening was not related to subsequent shortening of the radius or to distal radial deformity, providing evidence against the ulnar tether theory ${ }^{6}$. Alternatively, their results suggest that both the radius and the ulna exert proportional suppression of growth, and the loss of radial length is caused by involvement of the radius in the pathological process, and not by an ulnar tether. This speculative argument was supported by Ogden on the basis of a histological study of an osteochondroma in the fibula of a three-year-old girl, which showed that the osteochondroma invaded the epiphysis and had the potential to further invade the growth plate ${ }^{7}$. Masada et al. classified forearm deformities that were due to multiple osteochondromas and reported the outcome of several surgical procedures; these data showed that the bulk of osteochondromas themselves plays an important role in inducing radial bowing ${ }^{8}$. Therefore, forearm deformity may be the result of various etiologic factors associated with multiple osteochondromas (ulnar shortening, growth disturbance, and/or compression by the tumor itself).

Tumor excision is the most common surgical treatment, and this procedure can be combined with other surgical methods, including ulnar lengthening, radial osteotomy, and hemiepiphysiodesis, depending on the severity and pattern of the deformity ${ }^{3-58-13}$. However, the variety of combinations of surgical procedures has made it difficult to assess the effectiveness of each procedure for the correction of the deformity, leading to a long-standing controversy regarding the overall effectiveness of the excision of osteochondromas in controlling the progression of a deformity. Masada et al. suggested that a simple excision can prevent the progression of the disease and is effective in controlling radial bowing; however, Fogel et al., in a study of simple excision in ten patients, found that early excision of the osteochondroma alone did not improve the wrist deformity, although the procedure did reduce the rate of progression of ulnar shortening compared with the preoperative rate ${ }^{10}$.

The purpose of the current study was to investigate the effect of simple excision of osteochondroma(s) on the correction of the deformity, on the basis of the changes in radiographic parameters. Various patterns of deformity were classified radiographically according to the location of the tumor, and these were related to the etiologies of the deformity. We hypothesized that a forearm deformity can be defined by the tumor location and that the type of deformity influences the effectiveness of simple excision for the correction of the deformity.

\section{Materials and Methods}

Cifty-seven surgical procedures in twenty-five patients (thirty forearms) with multiple osteochondromas were performed in our department between 1983 and 2005. Excision of osteochondromas was performed in all patients. Accompanying procedures included ulnar lengthening (eighteen forearms), radial osteotomy (two forearms), and radial hemiepiphysiodesis (seven forearms). The ulnar lengthening was usually performed when the radial head was dislocated proximally in association with severe ulnar shortening, and radial osteotomy or radial hemiepiphysiodesis was performed when the lunate had slipped beyond the ulnar edge of the distal part of the radius with increased radial inclination of the distal end of the radius. The purpose of this study was to assess the effectiveness of tumor excision for the correction of a deformity; therefore, fourteen forearms in thirteen patients who had a 

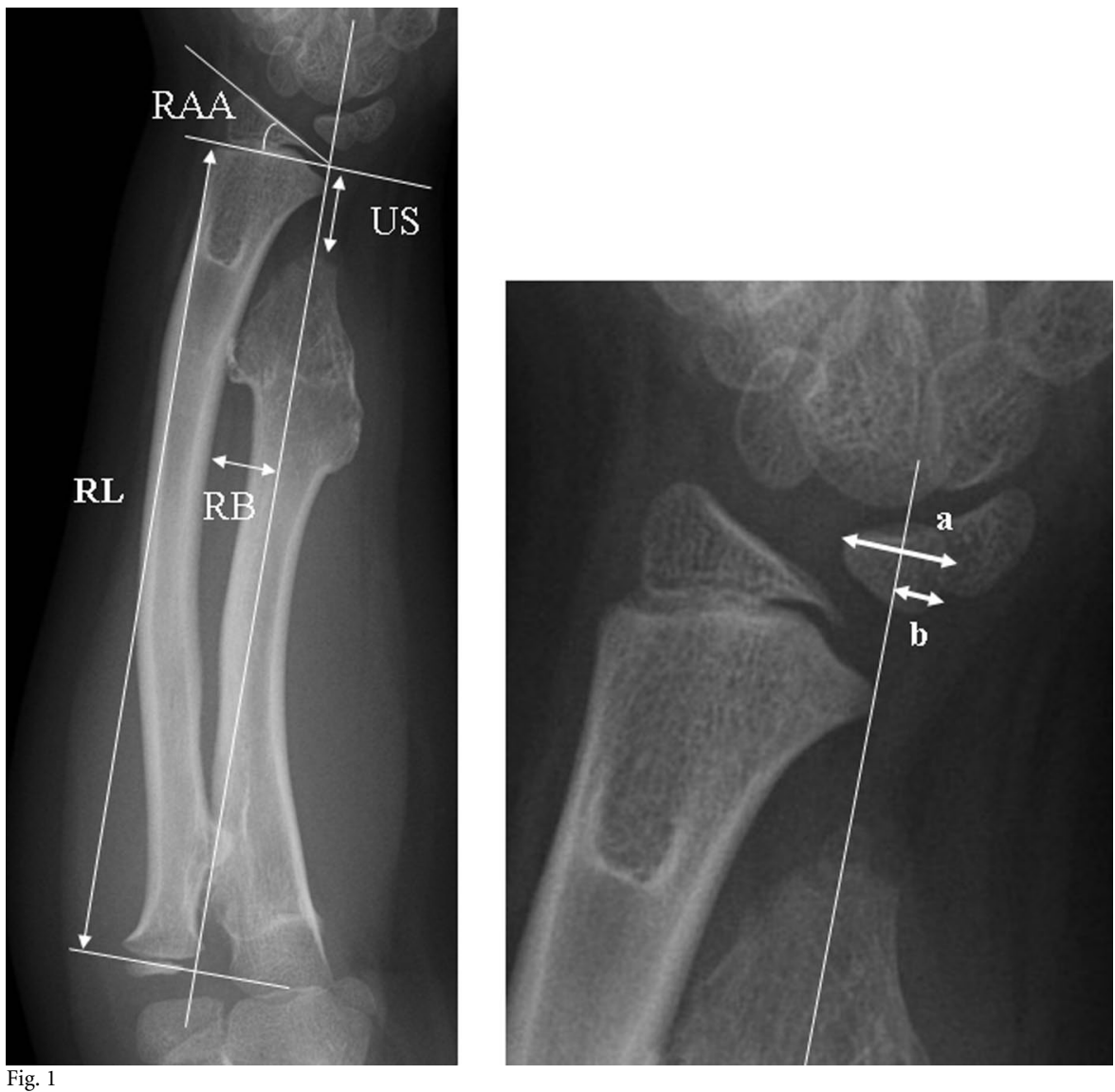

Radiographic measurement of ulnar shortening (US), radial bowing (RB), carpal slip, radial

length $(\mathrm{RL})$, and radial articular angle (RAA) (see text). The percentage of ulnar shortening =

US / RL $\times 100$; the percentage of radial bowing $=\mathrm{RB} / \mathrm{RL} \times 100$; and carpal slip $=\mathrm{b} / \mathrm{a} \times 100$.

simple tumor excision as an isolated procedure and were followed for more than twenty-four months were included in the study (Table I). Informed consent was obtained preoperatively from the patients and parents on the basis of the surgical indication as determined at our institution, and the patients returned for follow-up evaluation postoperatively. The patients included six boys and seven girls. In one patient, tumors were excised from both forearms. The average age at the time of surgery was 7.9 years (range, four to fourteen years), and the operation was performed when the patients were less than ten years old, except for two children who had surgery when they were twelve and fourteen years old. The average follow-up period was fifty-three months (range, twenty-four to ninetyseven months).

The forearm and wrist deformity were evaluated with use of plain anteroposterior radiographs according to the method reported by Burgess and Cates (Fig. 1) ${ }^{6}$. In this method, a linear axis is determined by a line connecting the ulnar borders of the distal and proximal physeal plates of the radius. Ulnar shortening is measured as the distance between the intersection of a line drawn perpendicularly at the level of the distal end of the ulna to the linear axis and the ulnar border of the distal physeal plate of the radius; the percentage of ulnar shortening is then calculated by dividing ulnar shortening by the length of the linear axis of the ulna. The radial articular angle is the angle between the linear axis of the radius and a line drawn along the distal articular surface of the radius. Radial bowing is defined as the greatest distance from the radial diaphysis to the linear axis of the radius and is expressed as the percentage of radial bowing, which is determined by dividing the radial bowing by the length of the linear axis. Carpal slip is determined by measuring the distance between the extreme ulnar side of the lunate and a continuation of the linear axis of the ulna, with this distance expressed as a percentage of the total length of the lunate. The extreme ulnar side of the lunate is defined as the contact point of a line drawn parallel to the linear axis of the lunate. In two fouryear-old patients (Cases 4 and 6), the lunate had not yet ossified and carpal slip was excluded from the evaluation.

Two patterns of deformity were defined on the basis of the location of the tumor. In the six forearms in Group 1, the osteochondroma was present only at the junction of the metaphysis and diaphysis of the distal aspect of the ulna, and it resulted in compression of the radius, which was mainly reflected in altered radial bowing (Figs. 2-A and 2-B). The deformity of the distal end of the radius in this group was usu- 
The JOURnal of BONE \& JOINT SURgERY • JBIS. ORG Volume 89-A · Number $6 \cdot$ June 2007
Tumor Location Affects the Results of Simple Excision For Multiple Osteochondromas in the Forearm ally minimal. In the eight forearms in Group 2, the tumors were present at both the distal end of the ulna and the ulnar side of the radius, and they were in contact with each other (Figs. 3-A and 3-B). The percentage of ulnar shortening and the percentage of radial bowing were less severe in Group 2 compared with Group 1. No patient in either group had dislocation of the radial head; however, one patient (Case 4) in Group 1 showed lateral subluxation of the radial head, with severe ulnar shortening.

Osteochondromas were excised completely under radiographic image control because there was no clearly identifiable border between the base of the tumor and the normal bone. Usually the normal bone was partly excised at the base of the tumor with great care taken to not injure the growth plate. In Group 1, the osteochondroma of the distal part of the ulna was approached between the extensor carpi ulnaris and the flexor carpi ulnaris. In Group 2, the osteochondroma of each bone was excised through two separate skin incisions in order to reduce the possibility of creating a synostosis between the distal end of the radius and the ulna after the excision ${ }^{12}$. The osteochondroma on the ulnar side of the distal part of the radius was exposed through a radiopalmar skin incision with detachment of the pronator quadratus from the radius.

We compared the groups in terms of the preoperative radiographic parameters using the Mann-Whitney test. To evaluate the effect of simple tumor excision on the correction of the deformity, the radiographic parameters before surgery and at the time of the final follow-up were compared with use of the paired t test and the groups were compared, with use of the Mann-Whitney test, with respect to the changes in each parameter after surgery. A p value of 0.05 was considered to be significant.

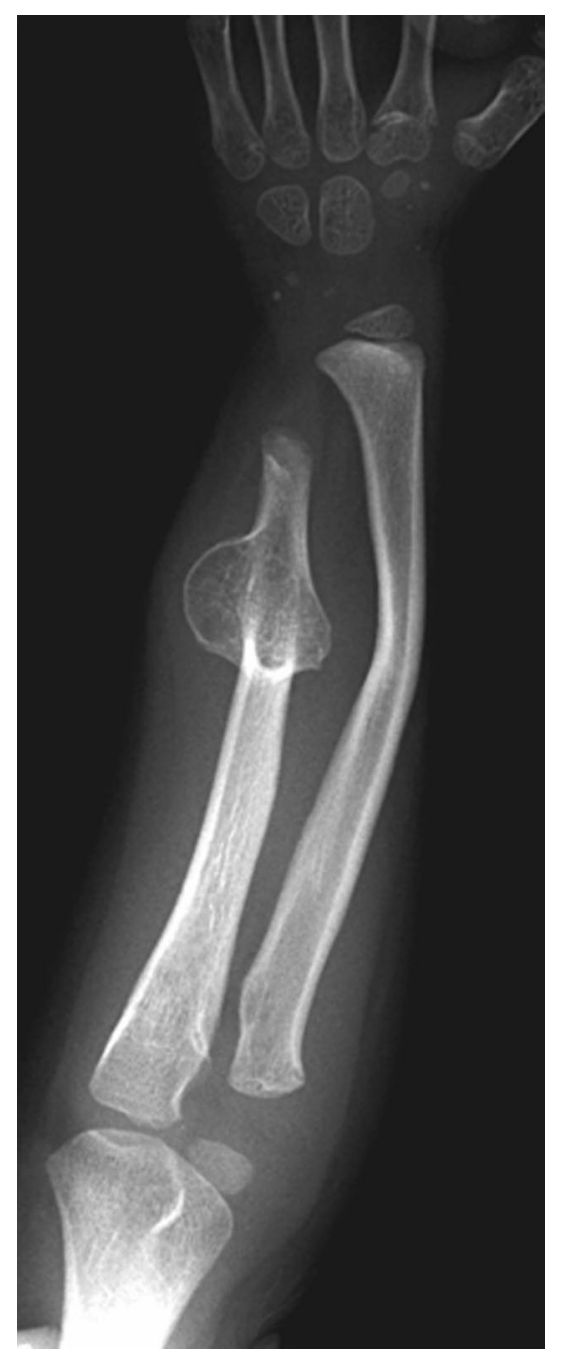

Fig. 2-A

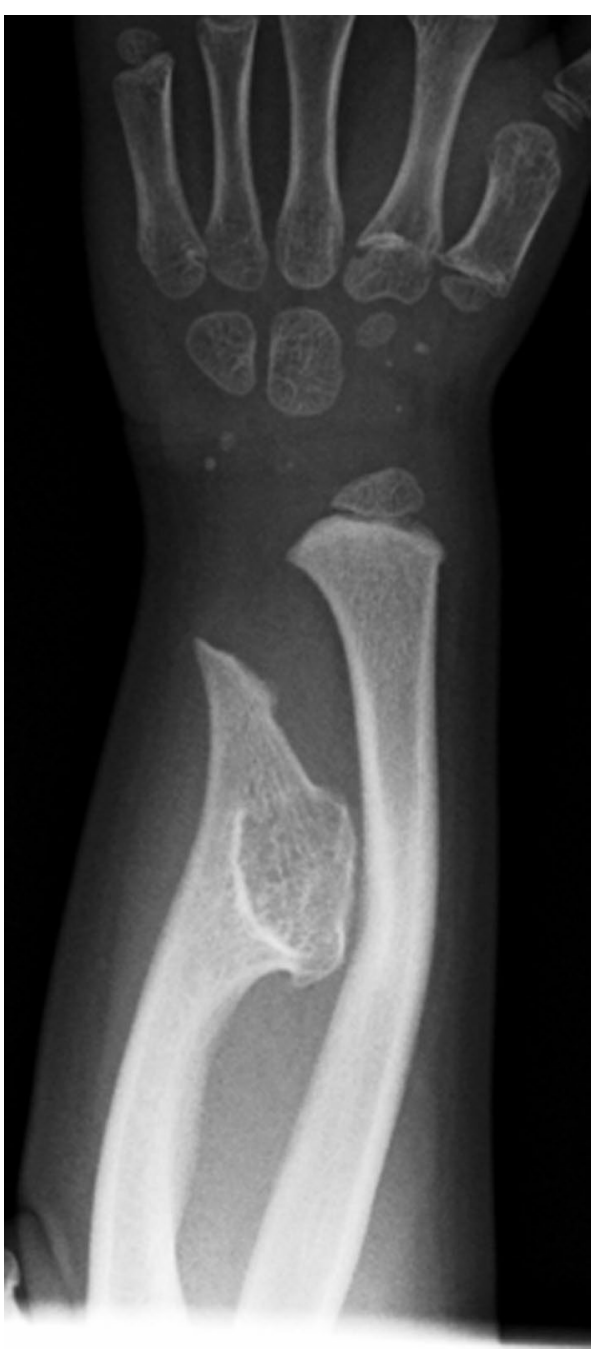

Fig. 2-B

Figs. 2-A through 2-D Case 4. A four-year-old girl in Group 1 who had a deformity of the left forearm. Figs. 2-A and 2-B Preoperative radiographs showing a tumor in the distal diaphysis of the ulna with compression of the radius. The percentage of ulnar shortening was $15.2 \%$, the percentage of radial bowing was $13 \%$, and the radial articular angle was $28^{\circ}$. 
The JOURnAL OF BONE \& JOINT SURGERY • JBIS. ORG VOlume 89-A · Number 6 · June 2007
Tumor Location Affects the Results of Simple Excision For Multiple Osteochondromas in the Forearm

\section{Results}

\section{Preoperative Findings}

D reoperative and postoperative radiographic parameters are shown in Table I. Groups 1 and 2 were compared with respect to these parameters. The average percentage of ulnar shortening, percentage of radial bowing, radial articular angle, and percentage of carpal slip were $8.3 \%, 10.2 \%, 30.5^{\circ}$, and $70.3 \%$, respectively, in Group 1 and $5.4 \%, 6.9 \%, 34.5^{\circ}$, and $71.6 \%$, respectively, in Group 2. Before surgery, the percentage of ulnar shortening and percentage of radial bowing were more severe in Group 1. A significant difference was recog- nized in the percentage of radial bowing $(\mathrm{p}=0.010)$. There were no apparent differences in radial articular angle or carpal slip between Groups 1 and 2 .

\section{Postoperative Findings}

There were no significant differences between Groups 1 and 2 in terms of age at the time of surgery (average, 6.9 and 8.7 years, respectively) or length of follow-up (fifty and fifty-six months, respectively).

The average changes in the radiographic parameters for all thirteen patients from before surgery to the final follow-up

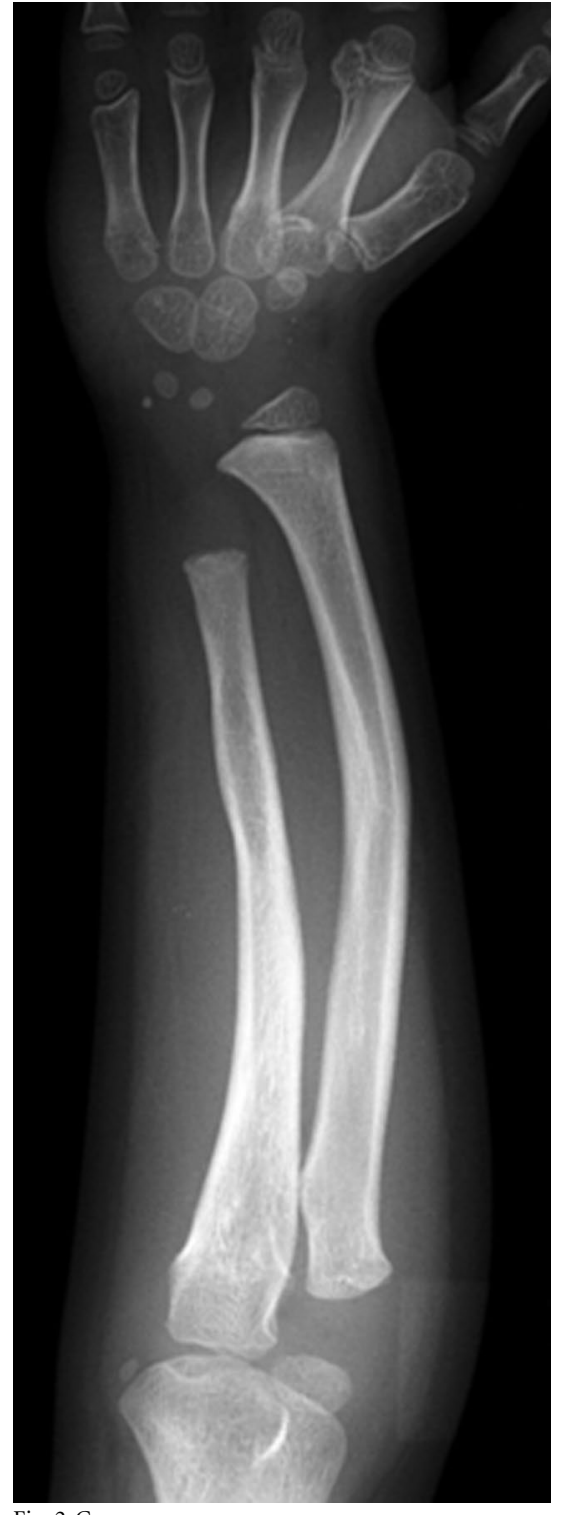

Fig. 2-C

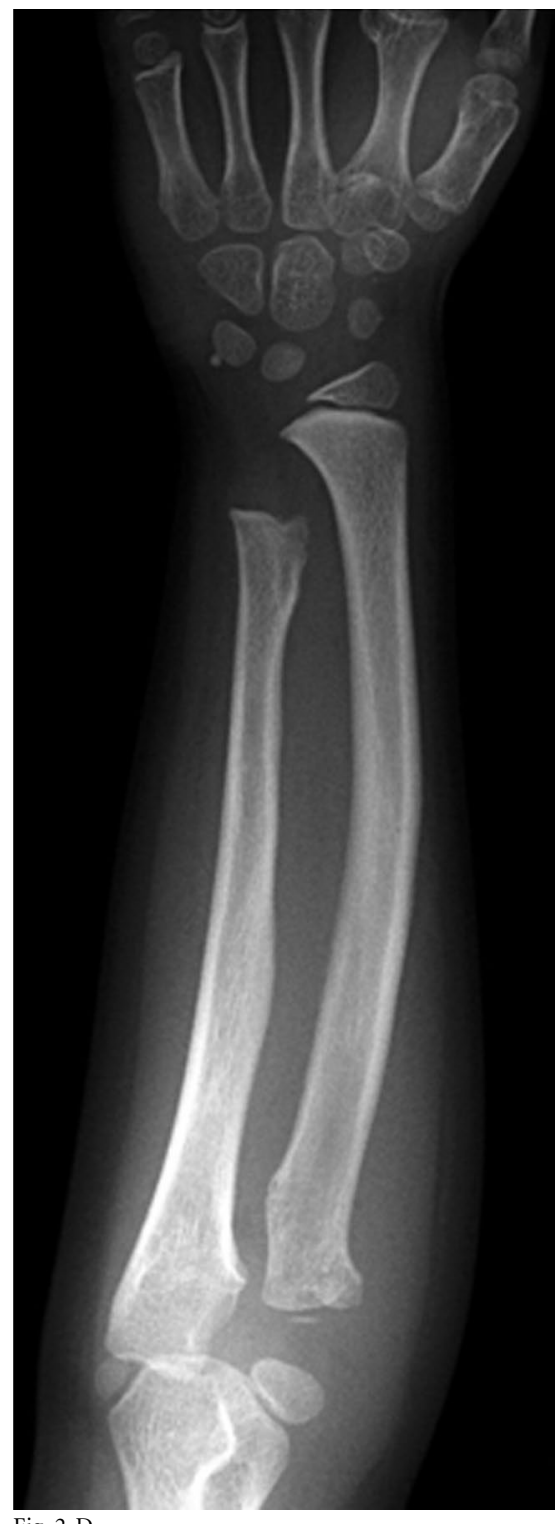

Fig. 2-D

Fig. 2-C Radiograph made twelve months postoperatively showing substantial remodeling of the bowed radius. Fig. 2-D Radiograph made twenty-seven months postoperatively showing that, although ulnar shortening remained, the percentage of ulnar shortening had improved to $11.8 \%$. Radial remodeling had proceeded further, and the percentage of radial bowing had improved to $8.1 \%$. The radial articular angle was unchanged from the preoperative value of $28^{\circ}$. 
The Journal of Bone \& JOINT SURGERY · JBJS.org VOLUME 89-A · Number $6 \cdot$ JUNE 2007
Tumor Location AfFects the Results of Simple Excision For Multiple Osteochondromas in the Forearm

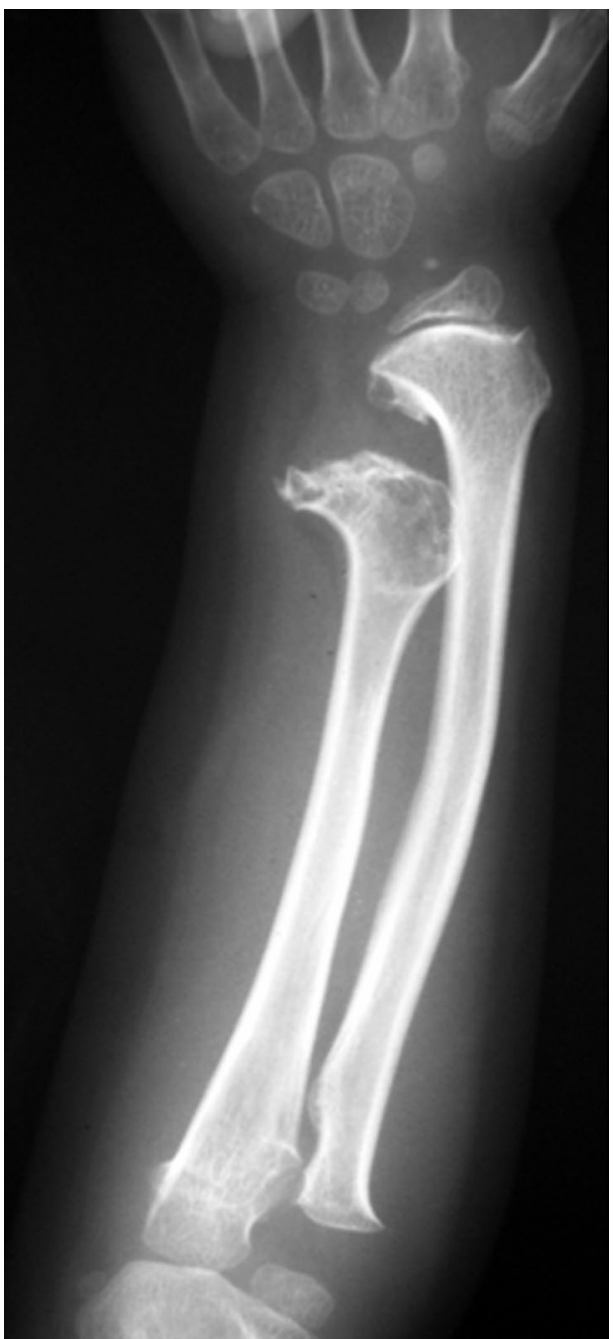

Fig. 3-A

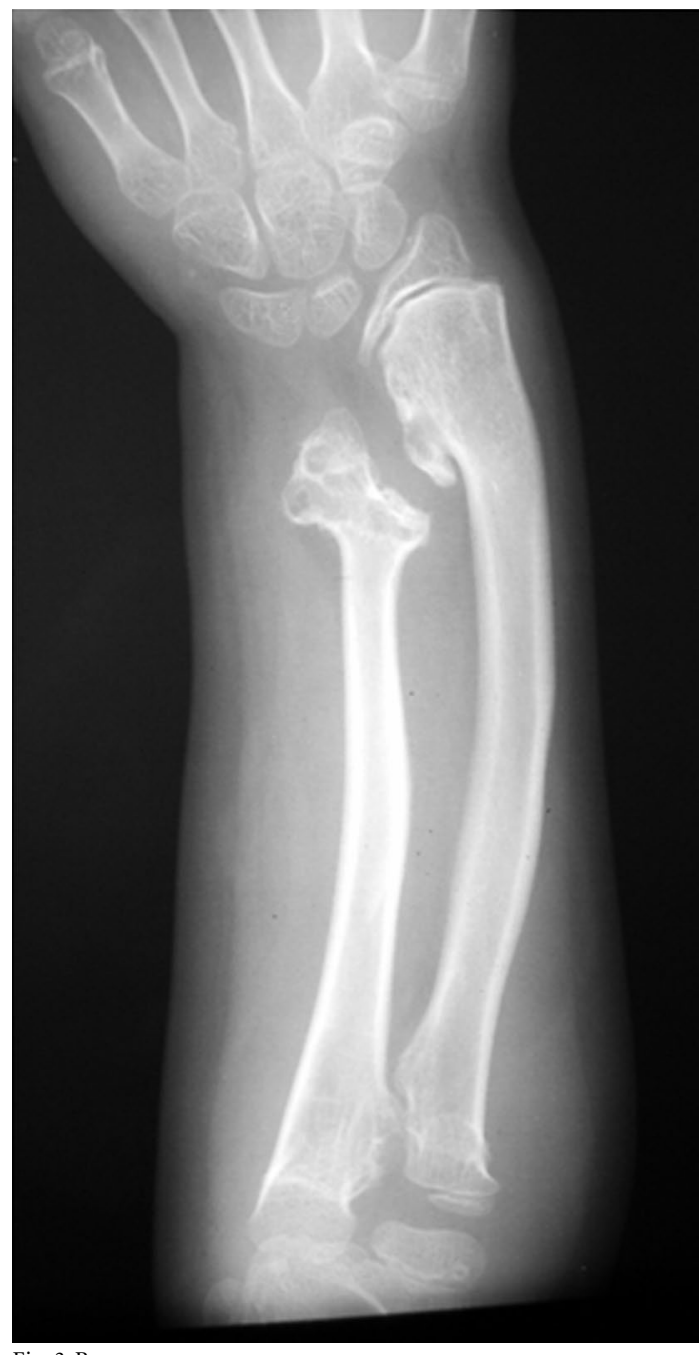

Fig. 3-B

Figs. 3-A and 3-B Case 7. A six-year-old boy in Group 2 who had a deformity of the left forearm. Fig. 3-A Radiograph showing osteochondromas in the metaphysis of the distal aspect of both the radius and ulna, which were in contact with each other. The percentage of ulnar shortening was $9.3 \%$, the percentage of radial bowing was also $9.3 \%$, the radial articular angle was $42^{\circ}$, and the amount of carpal slip was $90 \%$. Fig. 3-B Radiograph made forty-six months postoperatively showing the percentage of ulnar shortening and radial bowing were unchanged ( $8.3 \%$ and $9.4 \%$, respectively), but both the radial articular angle and the carpal slip had deteriorated to $63^{\circ}$ and $111.8 \%$, respectively. The tumors had recurred, and a growth disturbance of the ulnar side of the distal aspect of the radius was suspected.

were $6.7 \%$ to $6.1 \%$ of ulnar shortening, $32.8^{\circ}$ to $37.2^{\circ}$ in the radial articular angle, $8.3 \%$ to $7.7 \%$ of radial bowing, and $71.1 \%$ to $64 \%$ of carpal slip. With this small number of patients, none of these changes were significant.

\section{Changes in Radiographic Parameters After Surgery Ulnar Shortening (Fig. 4)}

The average percentage of ulnar shortening improved from $8.3 \%$ before surgery to $6.5 \%$ at the time of the final follow-up in Group 1 ( $\mathrm{p}=0.047)$, whereas no change was detected, with the numbers studied, in Group 2 (5.4\% to 5.8\%, respectively). The difference between the two groups with respect to the av- erage change in the percentage of ulnar shortening $(-1.8 \%$ in Group 1 and $+0.4 \%$ in Group 2) after surgery was significant $(\mathrm{p}=0.039)$. In Group 1, improvement of $>2 \%$ was obtained in three patients (Cases 2, 3, and 4) (Figs. 2-A through 2-D) and two patients (Cases 1 and 5) showed almost no change in the percentage of ulnar shortening. In contrast, one patient in Group 2 (Case 13) showed a deterioration in the percentage of ulnar shortening of $>2 \%$.

\section{Radial Bowing (Fig. 5)}

The mean percentage of radial bowing in Group 1 improved significantly from $10.2 \%$ before surgery to $7.3 \%$ at the time of 
The Journal of Bone \& JoInt Surgery · JBJS.org VOLUME 89-A · Number 6 · June 2007
Tumor Location Affects the Results of Simple Excision For Multiple Osteochondromas in the Forearm

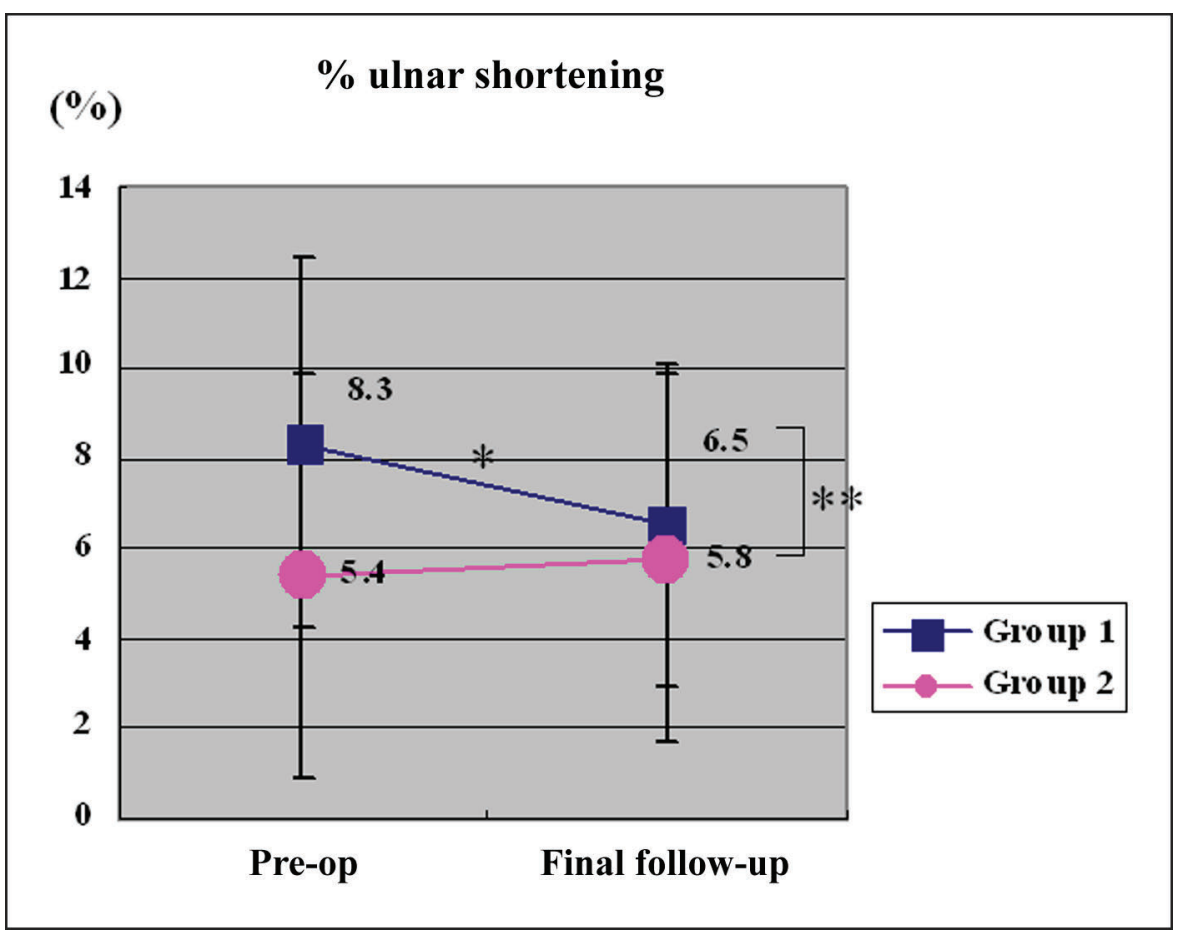

Fig. 4

Preoperatively, the average percentage of ulnar shortening was significantly more severe in Group 1 than in Group 2 (single asterisk; $p<0.05$ ). At the time of the final follow-up, it had significantly improved to an average of $6.5 \%$ in $\mathrm{Group} 1$ (single asterisk; $p<0.05$ ), whereas there was no change in Group 2 (average, 5.8\%). The difference in the postoperative change between the groups was significant (double asterisk; $p<0.05$ ).

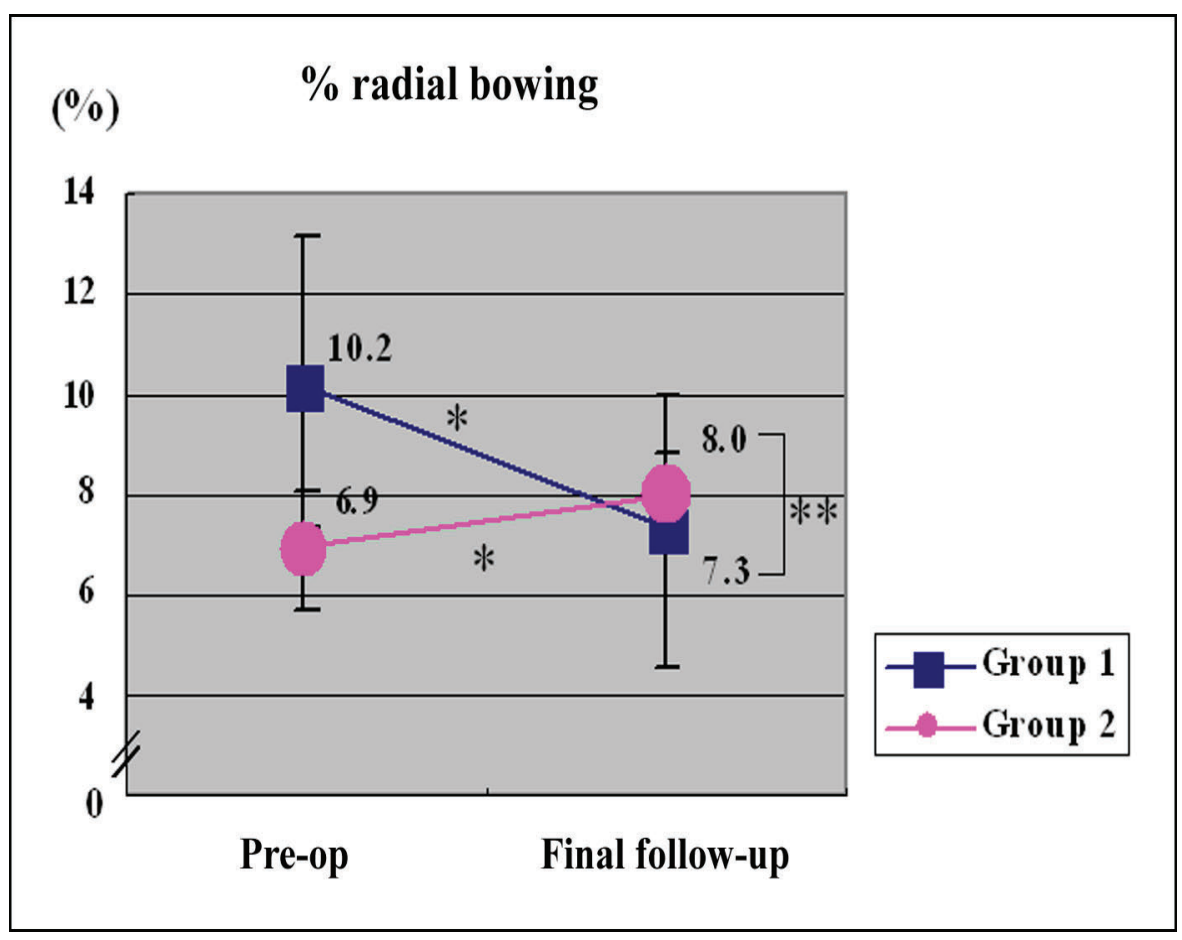

Fig. 5

Preoperatively, the average percentage of radial bowing was significantly more severe in Group 1 than in Group 2 ( $p=$ 0.010). At the time of final follow-up, it had significantly improved in Group 1, but had significantly deteriorated in Group 2 (single asterisk; $p<0.05$ for both). The difference between the groups was significant (double asterisk, $p<0.05)$. 


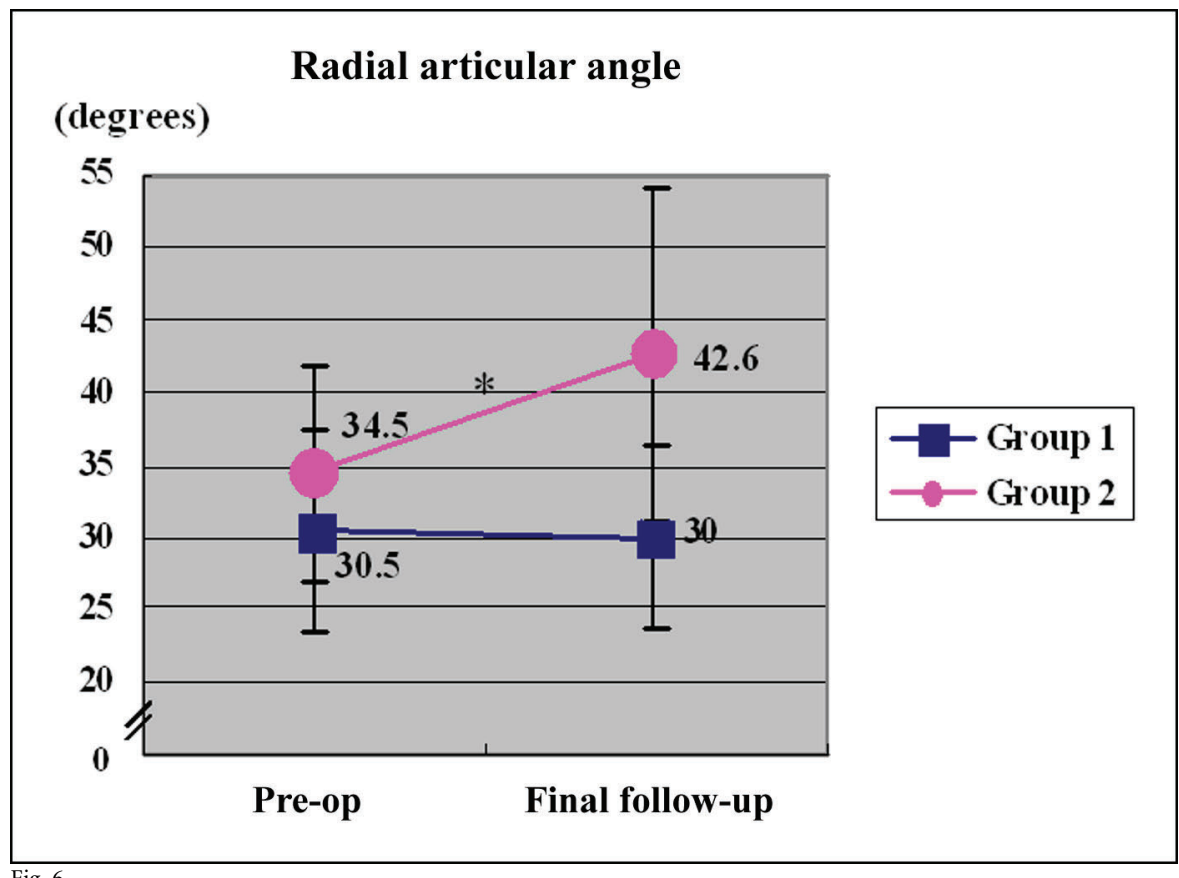

Fig. 6

At the time of final follow-up, the radial articular angle was unchanged in Group 1, but it had significantly deteriorated in Group 2 (asterisk; $p<0.05$ ).

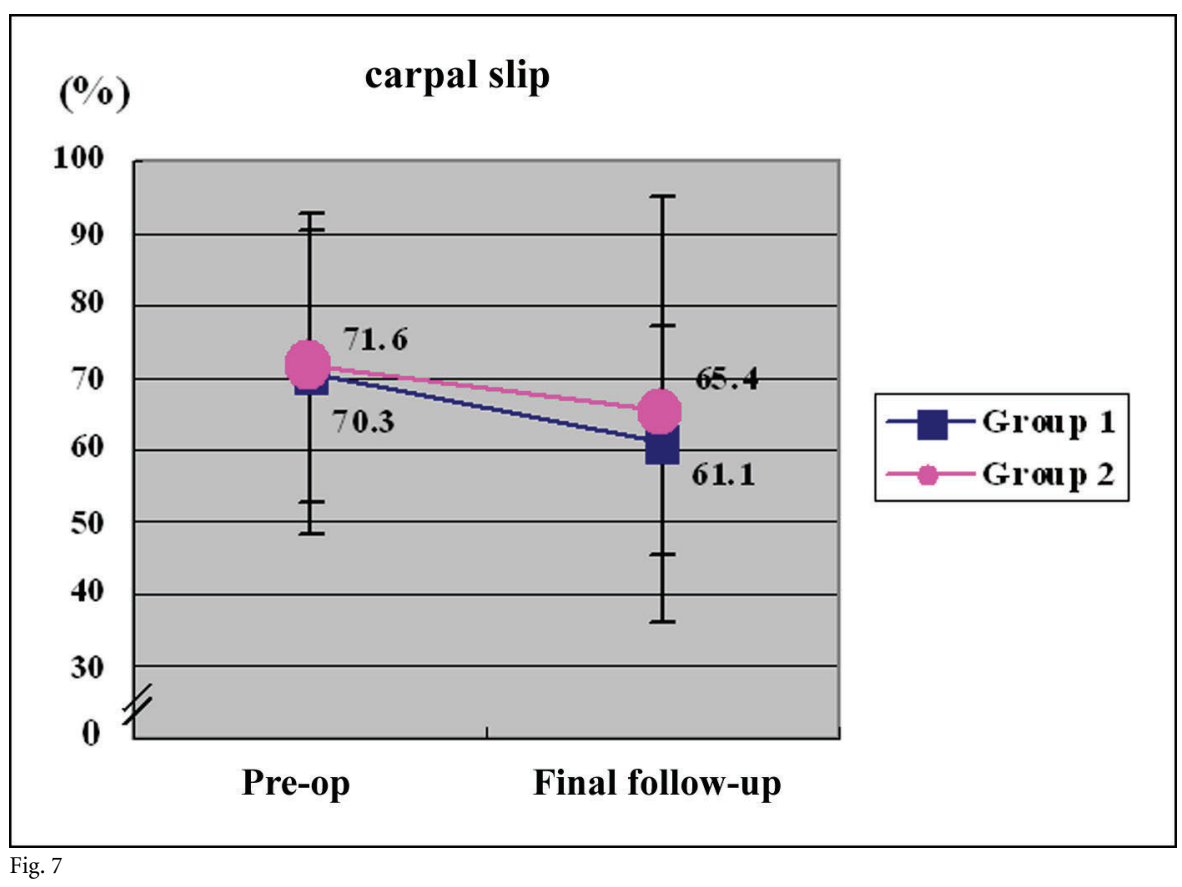

The amount of preoperative carpal slip showed no significant change at the time of final follow-up.

the final follow-up $(\mathrm{p}=0.010)$. Conversely, the mean percentage of radial bowing in Group 2 showed significant deterioration from $6.9 \%$ before surgery to $8.0 \%$ at the time of the final follow-up ( $\mathrm{p}=0.017$ ). The difference between the groups with respect to the average change after surgery $(-2.9 \%$ in Group 1 and $+1.1 \%$ in Group 2$)$ was also significant $(\mathrm{p}=0.002)$.
Radial Articular Angle (Fig. 6)

At the time of the final follow-up, the mean radial articular angle had not changed in Group $1\left(30.5^{\circ}\right.$ before surgery to $30.0^{\circ}$ at the time of the final follow-up), but it had significantly deteriorated from $34.5^{\circ}$ to $42.6^{\circ}$ in Group $2(\mathrm{p}=0.049)$. The difference between Groups 1 and 2 with respect to the 
The Journal of Bone \& JOINT SURGERY • JBIS. ORG Volume 89-A · Number $6 \cdot$ June 2007
Tumor Location Affects the Results of Simple Excision For Multiple Osteochondromas in the Forearm mean change after surgery $\left(-0.5^{\circ}\right.$ and $+8.1^{\circ}$, respectively) was not significant $(\mathrm{p}=0.106)$. In four patients (Cases $7,8,11$, and 12) in Group 2, the radial articular angle had increased by $\geq 10^{\circ}$ at the time of final follow-up compared with the preoperative value.

\section{Carpal Slip (Fig. 7)}

The average carpal slip in Groups 1 and 2 was $70.3 \%$ and $71.6 \%$, respectively, before surgery and $61.1 \%$ and $65.4 \%$, respectively, at the time of the final follow-up. In both groups, the average carpal slip showed slight improvement; however, in two patients (Cases 7 and 8) in Group 2, carpal slip increased severely and reached values of $>100 \%$ with accompanying increases in the radial articular angle at the time of the final follow-up (Fig. 3-B).

\section{Recurrence of the Tumor}

Some degree of tumor recurrence was recognized on the distal aspect of both the ulna and the radius in five patients (Cases 7 and 8 and, on the left side, in Cases 10, 12, and 13) in Group 2 and on the distal aspect of the ulna in two patients (Cases 2 and 6) in Group 1 at the time of the final follow-up. Recurrence was more frequent in the patients with the longer follow-up period. However, there were no clear differences in the radiographic parameters after surgery between the patients with and those without tumor recurrence in either group.

\section{Discussion}

Tn our patients with osteochondromas of the distal aspect of the forearm, the pattern of deformity differed according to the location of the tumor. In Group 1 (with the tumor in the metaphyseal-diaphyseal area and located far from the growth plate of the ulna), the percentage of ulnar shortening and the percentage of radial bowing were worse than those in Group 2, leading to compression of the radius. Radial bowing in the patients in Group 1 was, therefore, thought to be partly due to compression of the radius caused by the tumor, as well as to relative ulnar shortening. ${ }^{8}$. The radial articular angle was usually minimal in the patients in Group 1, although ulnar shortening was severe; this is inconsistent with the ulnar tether theory and in agreement with the conclusions of Burgess and Cates $^{6}$. Simple excision of the tumor resulted in improvement in the relative amounts of ulnar shortening and radial bowing in Group 1, indicating that radial bowing can be expected to correct by normal remodeling of the bowed radius, as well as through recovery of the ulnar growth rate after removal of the tumor, especially in younger patients with a greater potential for remodeling. In Group 1, there appeared to be little tethering effect between the distal aspects of the ulna and the radius; therefore, simple tumor excision should be enough to correct the forearm deformity when there is no dislocation of the radial head proximally.

The percentage of ulnar shortening in the patients in Group 2 was less severe than that in Group 1, which may reflect a growth disturbance of the distal aspect of the radius due to involvement by the tumor. Burgess and Cates described two patients with an increased radial articular angle in conjunction with positive ulnar variance ${ }^{6}$, in whom the radiographic findings showed the tumor to be on the ulnar side of the distal part of the radius. Masada et al. classified forearm deformity according to the location of the osteochondromas ${ }^{8}$. In that classification system, Type-III deformities are those in which the tumor is in the distal aspect of the radius and is accompanied by relative radial shortening.

The effectiveness of different surgical approaches to correct a forearm deformity due to multiple osteochondromas was reported by Fogel et al. ${ }^{10}$ and included the interesting case of a patient who had the same type of deformity as those in Group 2 in our study; that is, the tumors were on both the ulnar side of the distal aspect of the radius and the distal part of the ulna and were in contact with each other. After observation of that patient for eight years, the deformity of the wrist had progressed and radial hemiepiphysiodesis was performed to correct the deformity by retarding growth on the radial side of the distal part of the radius ${ }^{10}$. However, this procedure also resulted in a difference in forearm length of $2.3 \mathrm{~cm}$ compared with the contralateral forearm. In contrast to the findings in that patient, Wood et al. stated that if an osteochondroma is located on the radius, or if osteochondromas from both the radius and the ulna push against each other, only minimal deformity occurs ${ }^{5}$.

In the current study, the percentage of ulnar shortening and the percentage of radial bowing were less severe in the patients in Group 2 than in those in Group 1, which is consistent with the findings of Wood et al. ${ }^{5}$. However, we suspect that the ulnar aspect of the distal part of the radius is more susceptible to growth disturbance compared with other parts of the radius. When tumors are on both the ulnar side of the distal part of the radius and the distal aspect of the shortened ulna and are in contact with each other, a tethering effect between the tumors might occur in a longitudinal direction and result in an increasing radial articular angle and radial bowing with the passage of time because of a growth disturbance of the ulnar side of the distal aspect of the radius. Solomon described a reason for the valgus deformity of the distal part of the tibia when osteochondromas develop in both the distal part of the tibia and the distal aspect of the fibula ${ }^{2}$. He concluded that the valgus deformity was caused by a tethering effect of the shortened fibula and not by uneven growth of the tibial physis because the distal tibial physis remained in its normal horizontal orientation. However, in our patients in Group 2, the distal radial physis showed ulnar tilting (Fig. 3-B), which suggests a growth disturbance of the ulnar side of the distal radial physis.

In our study, simple excision of the tumor was unable to correct the deformity in the patients in Group 2, and the radial articular angle and the percentage of radial bowing showed significant increases. It is difficult to conclude whether simple tumor excision in the patients in Group 2 was effective for the correction of the deformity because of the high recurrence rate of the tumor in five of the eight forearms. Such recurrence of the tumor after excision has been 
THE JOURNAL OF BONE \& JOINT SURGERY • JBJS.ORG Volume 89-A · Number $6 \cdot$ June 2007
Tumor location Affects the Results of Simple Excision For Multiple Osteochondromas in the Forearm reported to be frequent. Shin et al., in a study involving twenty-two patients managed with simple excision of osteochondromas, noted that the recurrence rate was $53.8 \%$ in patients who were less than ten years old ${ }^{13}$. We think that the tethering effect and the growth disturbance of the affected portions persisted even after tumor excision irrespective of tumor recurrence in Group 2. This resulted in the deterioration of the radial articular angle and radial bowing. Actually, the percentage of radial bowing in three patients (Cases 8 [right side], 9, and 11) and the radial articular angle in one patient (Case 11) deteriorated, although there was no recurrence of the tumor. We recommend ulnar lengthening to completely release the tethering and to support the ulnar carpus when the radial articular angle and carpal slip are severe in patients with a Group-2 type of deformity.

In conclusion, the pattern of forearm deformity can be classified on the basis of the location of the tumor. This pattern is determined by the absence (Group 1) or presence (Group 2) of the tethering effect between the ulnar side of the distal aspect of the radius and the distal end of the ulna, and the ability of simple surgical excision of the tumor to correct deformity is influenced by this pattern of deformity. We note that the number of patients in the current study was small, and a more extensive study is necessary to confirm these conclusions.

Jun-ichi Ishikawa, MD

Norimasa Iwasaki, MD

Naoki Suenaga, MD

Akio Minami, MD

Department of Orthopaedic Surgery, Hokkaido University School of Medicine, Kita-15, Nishi-7, Kita-ku, Sapporo 060-8638, Japan. E-mail address for J. Ishikawa: ishijet77@yahoo.co.jp

Hiroyuki Kato, MD

Department of Orthopaedic Surgery, Shinshu University School of Medicine, 3-1-1 Asahi, Matsumoto, Nagano 390-8621, Japan

Fumio Fujioka, MD

Department of Orthopaedic Surgery, Nagano Prefectural Children's Hospital, 3100 Toyoshina, Azumino, Nagano 399-8288, Japan

\section{References}

1. Solomon L. Hereditary multiple exostosis. J Bone Joint Surg Br. 1963;45: 292-304.

2. Solomon L. Bone growth in diaphysial aclasis. J Bone Joint Surg Br. 1961;43:700-16.

3. Pritchett JW. Lengthening the ulna in patients with hereditary multiple exos toses. J Bone Joint Surg Br. 1986;68:561-5.

4. Siffert RS, Levy RN. Correction of wrist deformity in diaphyseal aclasis by stapling. Report of a case. J Bone Joint Surg Am. 1965;47:1378-80.

5. Wood VE, Sauser D, Mudge D. The treatment of hereditary multiple exostosis of the upper extremity. J Hand Surg [Am]. 1985;10:505-13.

6. Burgess RC, Cates $\mathrm{H}$. Deformities of the forearm in patients who have multiple cartilaginous exostosis. J Bone Joint Surg Am. 1993;75:13-8.

7. Ogden JA. Multiple hereditary osteochondromata. Report of an early case. Clin Orthop Relat Res. 1976;116:48-60.
8. Masada K, Tsuyuguchi $\mathrm{Y}$, Kawai $\mathrm{H}$, Kawabata H, Noguchi K, Ono K. Operations for forearm deformity caused by multiple osteochondromas. J Bone Joint Surg Br. 1989;71:24-9.

9. Ip D, Li YH, Chow W, Leong JC. Reconstruction of forearm deformities in multiple cartilaginous exostoses. J Pediatr Orthop B. 2003;12:17-21.

10. Fogel GR, McElfresh EC, Peterson HA, Wicklund PT. Management of deformities of the forearm in multiple hereditary osteochondromas. J Bone Joint Surg Am. 1984;66:670-80

11. Jiya TU, Pruijs JE, van der Eijken JW. Surgical treatment of wrist deformity in hereditary multiple exostosis. Acta Orthop Belg. 1997;63:256-61.

12. Peterson HA. Deformities and problems of the forearm in children with multiple hereditary osteochondromata. J Pediatr Orthop. 1994;14:92-100.

13. Shin EK, Jones NF, Lawrence JF. Treatment of multiple hereditary osteochondromas of the forearm in children: a study of surgical procedures. J Bone Joint Surg Br. 2006;88:255-60. 